\title{
Modelling Popularity Dynamics Based on YouTube Viewers and Subscribers
}

\author{
Mohammed Shahid Irshad \\ Department of Operational Research, \\ University of Delhi, Delhi 110007, India \\ E-mail: mohammedshaihd.irshad@gmail.com \\ Adarsh Anand \\ Department of Operational Research, \\ University of Delhi, Delhi 110007, India \\ Corresponding author: adarsh.anand86@gmail.com

\section{Marut Bisht} \\ Department of Management and Commerce, \\ Amity University, Dubai, United Arab Emirates \\ E-mail: mbisht@amityuniversity.ae \\ (Received May 22, 2018; Accepted August 17, 2019)
}

\begin{abstract}
There are plenty of domains as far as research in social media is concerned; Social Network, Video social media, image social media, research/ professional social media and so on. In the present work, we have focused on YouTube which is one of the pioneering amongst video social media. We have modeled the popularity dynamics of YouTube videos based on the information spread amongst the netizens and the number of subscribers for a particular video. An alternative approach to reach to our proposal has also been provided. In practice, the pace of the spread of information might vary because of various reasons such as quality of information, word of mouth, social causes etc. This transformation has been inculcated in the aforesaid modeling and results obtained on the view-count data sets of YouTube videos are very promising.
\end{abstract}

Keywords-Subscribers, Viewership rate, View-count, YouTube.

\section{Introduction}

Humans have evolved from Paleolithic to Mesolithic/ Neolithic to Bronze to Iron age. From being a hunter \& gatherer to a fully civilized society; humanity has progressed through different revolutions. Every revolution shapes the history and future of this very human civilization. There are various revolutions that have occurred in each and every part of this world; like agricultural revolution and industrial revolution. One such revolution is information technology revolution which connects each and every person and so on of this world. In communication system we have evolved from smoke signals to smartphones. Internet is a product of information technology revolution. Technical foundation of internet is laid by ARPANET (Advance Research Project Agency Network) which is a packet-switching network and first network to implement the TCP/IP protocol. ARPANET was initially funded by Advanced Research Projects Agency (ARPA) of the United States Department of Defense. Started as a defense project today, it is used by each and every individual who use a smartphone. With increase in the applications of internet its users known as "netizens", increased immensely. 
International Journal of Mathematical, Engineering and Management Sciences

Vol. 4, No. 6, 1508-1521, 2019

https://dx.doi.org/10.33889/IJMEMS.2019.4.6-119

One to one communication like in Electronic mails (E. Mail.) and chatting is solely dependent upon internet. With the launch of Social platforms like Facebook in early 2005 by Mark Zukerberg (Cheng et al., 2008) users could share their thoughts and emotions with the world with just a single post. Initially the thoughts were shared in the form of text but now images, audio files as well as video files can be shared to express views. As the video and image sharing comes into picture these social platforms becomes social media platforms. YouTube is one of the various video sharing platforms where a user creates video and posts it on its channel and viewers view it. Started as a dating site YouTube soon become a very popular video sharing site. As popularity and number of viewers grew the video uploaders started getting the share of revenue from YouTube, which lead to the commercialization of videos. It evolved from "expressing the thoughts and emotions" to "source of revenue and expression of art". As the local uploaders grew to fame the top entertainment companies like T-series, Vevo, Sony also join in for business purpose. They also have their own channels where they upload video as a product. The revenue model of YouTube is very simple they provide two type of content: paid and free. Paid don't show any advertisement and have some exclusive content. Whereas in free content a viewer has to watch advertisement before, in between or at the end of video. In this advertisement based revenue system; uploader also receives share from the revenue which prompts his/her to make video which generate more and more views on a video. YouTube also introduced various feature to generate it user base for example "Subscribe" button is introduced so that if a user subscribed a channel than whenever video is uploaded on that channel, user receives a notification. It also has like dislike button along with comment section that allows user to express its views on the video. But out of all feature view-count is the stuff in which uploader and YouTube is interested in. The impression of advertisement is directly proportional to the view-count of a video, which states that predicting the view-count is twofold perspective. For YouTube and uploaders, it provides a blue print of how much revenue is generated and for advertisers it provides a time frame when to advertise on a particular video.

\subsection{Literature Review}

Video is considered as a product and like a normal and tangible product it too has a life cycle (Cheng et al., 2008). Every product has its life cycle, which is measured through different ways. In case of YouTube videos to find the stage of the lifecycle in which the video is we concentrate of the view-count pattern of the video. Even virality of the video is also judged by the view-count pattern prompting researchers of various fields to study the blueprint of view-count. In recent years various researchers tried to visualize the view-count pattern through different methods and models. Yu et al. (2015) proposed that the video life cycle is divided into different popularity phases and predicted the view-count. Bauckhage et al. (2015) proposed SIR (susceptible, infected or recovered) epidemic modelling to predict the view-count of the view. Richier et al. (2014) proposed six different models to capture the view-count growth of fixed population (Non-viral) and growing population (viral) videos. Zhou et al. (2010); Portilla et al. (2015) studies the effect of recommendation system on the view-count of a video. Khan and Vong (2014) study the effect of other platforms (Facebook, twitter, Google+, etc.) on the view-count of the video on YouTube as we can share the YouTube on these social media platforms. In this paper, we have proposed a methodical approach to understand popularity dynamics of YouTube videos based on the information spread amongst netizens including the role of subscribers for particular videos. In practice, the pace of the spread of information might vary because of various reasons such as quality of information, word of mouth, social causes etc. The transformation has been made use while modeling the proposed view-count model. 
International Journal of Mathematical, Engineering and Management Sciences

Vol. 4, No. 6, 1508-1521, 2019

https://dx.doi.org/10.33889/IJMEMS.2019.4.6-119

The rest of the paper has been structured as follows: Section 2 discuss the building blocks of the proposed modelling framework. Notations are described in section 3. Section 4 discusses the Models formulation whereas Model Validation is discussed in section 5. Conclusion is presented in section 5 followed by references at the end

\section{Notations}

The notations used in this paper are explained as follows:

$V, V(t) \quad$ Mean number of views per instruction in the time interval $(0, t]$

$U, U(t) \quad$ Cumulative number of users at time $t$ and $\frac{d}{d t} U(t)=u(t)$

$N \quad$ Potential number of viewers

$v_{1} \quad$ Rate at which video is watched through external influence.

$v_{2} \quad$ Rate at which video is viewed under the influence of word of mouth.

$\beta, r, R \quad$ Constants

\section{The Building Blocks of the Proposed Modelling Framework}

\subsection{The View Count Model}

Aggrawal et al. (2018a) presented an analogy from marketing science and proposed a framework which characterized the literary theory in terms of the view count. Their viewership computational model considered the association of two types of viewers in contributing to the overall view count. In their approach, they assumed viewership to be initiated by certain number of viewers after the launch of the video in the internet market. Furthermore, they assumed the rate of viewing any video at a given time to comprise of two components that administrate the viewing process; the first factor constitute the videos watched through external influence with an impact rate $v_{1}$ and the second factor represents the additional number of viewers who watch a video and under the influence of word of mouth (with rate $v_{2}$ ). Their View Count Process can be modelled in the following manner:

$\frac{d V(t)}{d t}=v_{1}(N-V(t))+v_{2} \frac{V(t)}{N}(N-V(t))$

Here $v_{1}$ represents the fraction of all viewers who are neoteric. The product $v_{2} / N$ times $V(T)$ reflects the pressure operating on followers as the number of early viewer's increases. After solving the equation (1) the closed form solution can be obtained as follows:

$V(t)=N\left(\frac{1-e^{-\left(v_{1}+v_{2}\right) t}}{1+\frac{v_{2}}{v_{1}} e^{-\left(v_{1}+v_{2}\right) t}}\right)$ 
International Journal of Mathematical, Engineering and Management Sciences

Vol. 4, No. 6, 1508-1521, 2019

https://dx.doi.org/10.33889/IJMEMS.2019.4.6-119

Their model so obtained had the ability to act as a forecasting tool that can help the firm in knowing the manner in which a video is able to generate popularity in terms of view count.

\subsection{An Alternate Formulation of the Aggrawal et al. (2017a) Modeling Framework}

We assumed the viewers who are a neoteric for use video may be a follower for another video. Furthermore, a follower might be exposed to medium through external influence. Hence makers use analogy from marketing system; it can be interpreted that as and when knowledge about the video grows with time, attributing it to the distinct category as defined by Aggarwal et al. (2018a) may be considered. However, due to the inherited flexibility of the model, it can be alternatively derived using a logistic tine dependent form for viewership. Therefore, equation (1) can be rewritten as:

$\frac{d V(t)}{d t}=(r(t))(N-V(t))$

As discussed above, we assume a logistic time dependent form for $r(t)$ to capture the aforesaid flexibility and so equation (3), can be represented as follows:

$\frac{d V(t)}{d t}=\left(\frac{r}{1+R \cdot e^{-r . t}}\right)(N-V(t))$

Where, $r$ is rate of viewership and $R$ represents the learning parameter with the initial condition at $t=0, V(\mathrm{t})=0$, we get:

$V(t)=N\left(\frac{1-e^{-r . t}}{1+R \cdot e^{-r . t}}\right)$

By substituting $r=v_{1}+v_{2}$ and $R=\frac{v_{2}}{v_{1}}$. it can be seen that Eq. (5) and Eq. (2) are identical. The S-shape in the cumulative curve is created by the logistic function of $r(t)$. The parameters in $r(t)$ represents external and internal influence factors and are amenable to further interpretations.

In the following section, we present some notations and assumptions that shall be required to build our integrated view count model.

\section{Model Formulation}

In this section, we propose to estimate the number of view counts for a particular video. It is done by establishing functional relationship between intensity of video surfed and the number of viewers of the video. To the best of our knowledge, such an approach has been attempted for the first time in the field of social media analysis. Several quantitative measures of growth in the popularity of a video have been proposed in the literature (Aggrawal et al., 2018b). 
International Journal of Mathematical, Engineering and Management Sciences

Vol. 4, No. 6, 1508-1521, 2019

https://dx.doi.org/10.33889/IJMEMS.2019.4.6-119

In the present work, an attempt has been made to model the view count growth linking it with the number of users which in turn depends on the number of subscribers. Therefore, the mean value function of view count process for the present situation can be developed based upon the following differential equation:

$$
\frac{d V(t)}{d t}=\left(\frac{d V(t)}{d U(t)}\right)\left(\frac{d U(t)}{d t}\right)
$$

In the above expression, $V$ and $U$ are differentiable functions of time but are explicitly dependent upon $U$ and $t$ respectively. The model to be proposed in this paper gives functional form to each term occurring within parenthesis in equation (7) based on the following assumptions:

Assumption 1: The view count rate at any time during its phase is dependent upon the number of viewers remaining in the system as well on the number of viewers who have already contributed in the view count process.

Using the concept given by Aggrawal et al. (2018a), we in this paper have presented a view count model that depends on the number of user (Subscriber). Thereby, the first part of equation (7) can be modelled as follows:

$$
\frac{d V(t)}{d U(t)}=\left(v_{1}+v_{2} \frac{V(t)}{N}\right)(N-V(t))
$$

\section{Assumption 2: The subscribers with respect to time of a channel follow power function.}

Power function has been explicitly used to describe the growth of user population in various industries (Singh et al., 2010). We make use of the same analogy to read the number of view counts based on number of subscribers of the channel and let the second component of expression (7) be defined as a power function of usage time. Thereby we take the following expression for the understanding

$\frac{d U(t)}{d t}=v_{3} t^{\beta}$

The function $U(t)$ can correctly describe the users' growth in terms of a slow start but gain in growth rate, a constant addition of users, and a big beginning and tail off in the usage rate. Here, $\beta \geq 0$ and if $\beta=0, \frac{d U(t)}{d t}=v_{3}$ (i.e. a constant), Substituting Eq. (8) and Eq. (9) in Eq. (7) we have:

$\frac{d V(t)}{d t}=\left(v_{1}+v_{2} \frac{V(t)}{N}\right) \cdot(N-V(t)) \cdot v_{3} \cdot t^{\beta}$ 
International Journal of Mathematical, Engineering and Management Sciences

Vol. 4, No. 6, 1508-1521, 2019

https://dx.doi.org/10.33889/IJMEMS.2019.4.6-119

It is a first order differential equation. Solving it with the initial condition $V(0)=0$; we get:

$$
V(t)=N\left(\frac{1-\exp \left\{\left(-\frac{1}{\beta+1}\right) \cdot v_{3}\left(v_{1}+v_{2}\right) \cdot t^{\beta+1}\right\}}{1+\frac{v_{2}}{v_{1}} \cdot \exp \left\{\left(-\frac{1}{\beta+1}\right) \cdot v_{3}\left(v_{1}+v_{2}\right) \cdot t^{\beta+1}\right\}}\right)
$$

As discussed in section 2 of the paper, we can obtain the resultant expression as obtained in equation (11) using the alternative approach. For this, we make use of equation (3) and redesign the differential equation as per our requirement by taking the following functional form of $r(t)$ :

$$
r(t)=\frac{r . t^{\beta}}{1+R \cdot \exp \left(-\frac{1}{\beta+1} \cdot r . t^{\beta+1}\right)}
$$

The expression in equation (12) represents a power logistic function and on solving using the initial set of conditions we get the same expression as obtained in equation (11).

$V(t)=N\left(\frac{1-\exp \left\{\left(-\frac{1}{\beta+1}\right) \cdot r \cdot t^{\beta+1}\right\}}{1+R \cdot \exp \left\{\left(-\frac{1}{\beta+1}\right) \cdot r \cdot t^{\beta+1}\right\}}\right)$

Here, $r=v_{3}\left(v_{1}+v_{2}\right)$ and $R=\left(v_{2} / v_{1}\right)$ and if we take $\beta=0$; the expression reduces to the model proposed by Aggrawal et al. (2018a).

\subsection{Changed Point Based Modelling Framework}

Change point is a very well-known and fundamentally accepted concept in management discipline. According to this concept, the adoption rate may not be smooth but might change at some time point because of many reasons. This concept holds good for understanding the rate of viewership in social media analysis. New videos are launched under specific internet market conditions to meet the demand of a certain section of society, known as target population. But the viewership process may get affected by many factors such as user's interest, popularity of the video, expectations and satisfaction level of the viewer, recommendations from peers and so on. It is also affected by change in internet market conditions such as entry/exit of competitive video from the market, launch of better or new video on the same or different channel. There are many more factors like position of the video in the search list, recommendation by the uploader, reviews, positive or negative word of mouth of the video and virality etc. which can alter viewership behavior that can bring in the concept of change point in social media analysis. Considering $\tau$ being the change point, the rate of viewership can be expressed before and after the change point in the following manner: 
International Journal of Mathematical, Engineering and Management Sciences

Vol. 4, No. 6, 1508-1521, 2019

https://dx.doi.org/10.33889/IJMEMS.2019.4.6-119

$$
r(t)=\left\{\begin{array}{cc}
\frac{r_{1} \cdot t^{\beta}}{1+R_{1} \cdot \exp \left(-\frac{1}{\beta+1} \cdot r_{1} \cdot t^{\beta+1}\right)} & t \leq \tau \\
\frac{r_{2} \cdot t^{\beta}}{1+R_{2} \cdot \exp \left(-\frac{1}{\beta+1} \cdot r_{2} \cdot t^{\beta+1}\right)} & t>\tau .
\end{array}\right.
$$

Here, $r_{l}$ and $r_{2}$ are viewership rates before and after change point. The view count process under the concept of change point can thus be modeled as

$$
\frac{d V(t)}{d t}=\left\{\begin{array}{l}
\frac{r_{1} \cdot t^{\beta}}{1+R_{1} \cdot \exp \left(-\frac{1}{\beta+1} \cdot r_{1} \cdot t^{\beta+1}\right)}(N-V(t)) \\
\frac{r_{2} \cdot t^{\beta}}{1+R_{2} \cdot \exp \left(-\frac{1}{\beta+1} \cdot r_{2} \cdot t^{\beta+1}\right)}(N-V(t))
\end{array}\right.
$$

After solving equation (13), we get

$$
V(t)=\left\{\begin{array}{l}
N \cdot\left(1-\left(\frac{1+R_{1}}{1+R_{1} \cdot \exp \left\{-\left(\frac{1}{\beta+1}\right) \cdot r_{1} \cdot t^{\beta+1}\right\}}\right) \cdot \exp \left\{-\left(\frac{1}{\beta+1}\right) \cdot r_{1} \cdot t^{\beta+1}\right\}\right) \text { for } 0 \leq t \leq \tau \\
N \cdot\left[1-\left(\left(\left(\frac{1+R_{1}}{\left.1+R_{1} \cdot \exp \left\{-\left(\frac{1}{\beta+1}\right) \cdot r_{1} \cdot t^{\beta+1}\right\}\right)}\right) \cdot\left(\frac{1+R_{2} \cdot \exp \left\{-\left(\frac{1}{\beta+1}\right) \cdot r_{2} \cdot \tau^{\beta+1}\right\}}{\left.\left.1+R_{2} \cdot \exp \left\{-\left(\frac{1}{\beta+1}\right) \cdot r_{2} \cdot t^{\beta+1}\right\}\right)\right)}\right)\right]\right.\right. \\
\left.\left.\exp \left\{-\left(\frac{1}{\beta+1}\right) \cdot\left(r_{1} \cdot \tau^{\beta+1}+r_{2} \cdot\left(t^{\beta+1}-\tau^{\beta+1}\right)\right)\right\}\right)\right]
\end{array}\right.
$$

If we take $\beta=0$ and $\tau=0$; the model reduces to the model proposed by Aggrawal et al. (2018a).

\section{Model Validation}

The three proposed models as per the scenario described are:

$$
\begin{array}{ll}
\text { Model I } \quad V(t)=N\left(\frac{1-e^{-r . t}}{1+R \cdot e^{-r . t}}\right) \\
\text { Model II } \quad V(t)=N\left(\frac{1-\exp \left\{\left(-\frac{1}{\beta+1}\right) \cdot r \cdot t^{\beta+1}\right\}}{1+R \cdot \exp \left\{\left(-\frac{1}{\beta+1}\right) \cdot r \cdot t^{\beta+1}\right\}}\right)
\end{array}
$$


International Journal of Mathematical, Engineering and Management Sciences

Vol. 4, No. 6, 1508-1521, 2019

https://dx.doi.org/10.33889/IJMEMS.2019.4.6-119

Model III $\left.V(t)=\left\{\begin{array}{r}N \cdot\left(1-\left(\frac{1+R_{1}}{\left.1+R_{1} \cdot \exp \left\{-\left(\frac{1}{\beta+1}\right) \cdot r_{1} \cdot t^{\beta+1}\right\}\right)}\right) \cdot \exp \left\{-\left(\frac{1}{\beta+1}\right) \cdot r_{1} \cdot t^{\beta+1}\right\}\right) \quad \text { for } 0 \leq t \leq \tau \\ N \cdot\left[1-\left(\left(\frac{1+R_{1}}{1+R_{1} \cdot \exp \left\{-\left(\frac{1}{\beta+1}\right) \cdot r_{1} \cdot t^{\beta+1}\right\}}\right) \cdot\left(\frac{1+R_{2} \cdot \exp \left\{-\left(\frac{1}{\beta+1}\right) \cdot r_{2} \cdot \tau^{\beta+1}\right\}}{\left.1+R_{2} \cdot \exp \left\{-\left(\frac{1}{\beta+1}\right) \cdot r_{2} \cdot t^{\beta+1}\right\}\right)}\right)\right) \cdot\right] \\ \left.\exp \left\{-\left(\frac{1}{\beta+1}\right) \cdot\left(r_{1} \cdot \tau^{\beta+1}+r_{2} \cdot\left(t^{\beta+1}-\tau^{\beta+1}\right)\right)\right\}\right)\end{array}\right)\right]$ for $t>\tau$

Model I is the alternate formulation of Aggarwal et al. (2018a) where we consider that the total the knowledge of the videos grow via word of mouth or other factors with time which creates a cumulative cure of S-shape. Model II deals with the effect of subscriber on the view-count of the video. We know that all the subscribers a channel of YouTube receives notification if video is uploaded on the YouTube. In this case we consider that subscriber follows power function with respect to time and view-count is dependent on number of subscribers. Model III deals with the change of behaviour of view-count by the viewers after certain point of time. As subscribers are getting notifications the initial rate of viewing is high but with time the almost all the subscribers watch the video and view-count totally depends on the word of mouth or other external factors. At that time the rate of change in view-count changes and that time is considered as change-point. In Model III we consider the effect of change point with subscribers on the view-count of a video. For the integrity of models they are tested on various YouTube video's view-count datasets.

\subsection{Description of Data Sets}

The view-count data of 6 videos is collected on 12 hourly bases for approximately 36 days. The YouTube address links of the 6 videos whose results are presented in this paper is shown in Table 1. Parameter estimation for all three models has been adjudged by using the technique of NonLinear Square Method. In this study, statistical software package SPSS (Statistical Package for Social Sciences) Non-Linear Regression Models have been used to estimate the parameters for the above defined three models (Anand and Bansal, 2016). Table 2-4 represents the estimated parameters of the data.

Table 1. YouTube address and category of videos

\begin{tabular}{|c|l|l|}
\hline Data Set & Youtube Address Link & Category \\
\hline DS 1 & "https://www.youtube.com/watch?v=PUIzmbxQ7O0" & Song \\
\hline DS 2 & "https://www.youtube.com/watch?v=Q6dsRpVyyWs" & Specification Video \\
\hline DS 3 & "https://www.youtube.com/watch?v=isQ5Ycie73U” & Movie Trailer \\
\hline DS 4 & "https://www.youtube.com/watch?v=r6FxROAHJH4" & Movie Trailer \\
\hline DS 5 & "https://www.youtube.com/watch?v=oB94lvJbETE" & Song \\
\hline DS 6 & "https://www.youtube.com/watch?v=GnORm4yR7pg" & Song \\
\hline
\end{tabular}


International Journal of Mathematical, Engineering and Management Sciences

Vol. 4, No. 6, 1508-1521, 2019

https://dx.doi.org/10.33889/IJMEMS.2019.4.6-119

Table 2. Parameter estimates of Model I

\begin{tabular}{|c|c|c|c|c|c|c|}
\hline Parameter & DS 1 & DS 2 & DS 3 & DS 4 & DS 5 & DS 6 \\
\hline$N$ & 2262.124 & 20295.33 & 3038.96 & 5626.885 & 20399.7 & 20399.7 \\
\hline$\beta$ & 8.485 & 0.278 & 0.01 & 0.228 & 0.01 & 0.01 \\
\hline$b$ & 0.211 & 0.339 & 0.081 & 0.102 & 0.134 & 0.134 \\
\hline
\end{tabular}

Table 3. Parameters estimates of Model II

\begin{tabular}{|c|c|c|c|c|c|c|}
\hline Parameter & DS 1 & DS 2 & DS 3 & DS 4 & DS 5 & DS 6 \\
\hline$N$ & 2259.924 & 20280.56 & 3033.881 & 5424.256 & 20384.6 & 1275.887 \\
\hline$\beta$ & 0.335 & 0.01 & 0.01 & 0.01 & 0.01 & 0.01 \\
\hline$K$ & 0.691 & 0.01 & 0.01 & 0.01 & 0.01 & 0.01 \\
\hline$B$ & 0.025 & 0.294 & 0.08 & 0.101 & 0.133 & 0.112 \\
\hline
\end{tabular}

Table 4. Parameter estimates of Model III

\begin{tabular}{|c|c|c|c|c|c|c|}
\hline Parameter & DS 1 & DS 2 & DS 3 & DS 4 & DS 5 & DS 6 \\
\hline$\beta_{1}$ & 0.253 & 0.01 & 0.01 & 0.01 & 0.01 & 0.01 \\
\hline$\beta_{2}$ & 0.99 & 0.01 & 0.01 & 0.01 & 0.01 & 0.01 \\
\hline$b_{1}$ & 0.054 & 0.238 & 0.061 & 0.093 & 0.119 & 0.103 \\
\hline$b_{2}$ & 0.075 & 0.224 & 0.043 & 0.086 & 0.095 & 0.088 \\
\hline$k$ & 0.308 & 0.01 & 0.01 & 0.01 & 0.01 & 0.01 \\
\hline$N$ & 2264.41 & 20380.26 & 3291.831 & 5485.437 & 20753.61 & 1297.632 \\
\hline
\end{tabular}

The performance analysis of the proposed models is measured by using the common goodness of fit criteria as MSE (Mean Square Error), RMPSE, $\mathrm{R}^{2}$ (Coefficient of determination), Bias and Variation. The value of these comparison criteria's shown in Table 5, confirming the robustness of the approach. 
International Journal of Mathematical, Engineering and Management Sciences

Vol. 4, No. 6, 1508-1521, 2019

https://dx.doi.org/10.33889/IJMEMS.2019.4.6-119

Table 5. Comparisons parameter of All Data Sets

\begin{tabular}{|c|c|c|c|}
\hline DS 1 & Model I & Model II & Model III \\
\hline R-Square & 0.992 & 0.992 & 0.992 \\
\hline Variance & 60.5024 & 59.48055 & 57.85862 \\
\hline Bias & 2.920847 & 1.633583 & 0.000375 \\
\hline M.S.E. & 3575.531 & 3472.58 & 3321.471 \\
\hline R.M.P.S.E & 60.57286 & 59.50297 & 57.85862 \\
\hline DS 2 & Model I & Model II & Model III \\
\hline R-Square & 0.894 & 0.932 & 0.946 \\
\hline Variance & 810.0245 & 655.5477 & 577.8968 \\
\hline Bias & -34.9428 & -21.1103 & -0.00817 \\
\hline M.S.E. & 646646.2 & 423818.8 & 330606.5 \\
\hline R.M.P.S.E & 810.7779 & 655.8876 & 577.8968 \\
\hline DS 3 & Model I & Model II & Model III \\
\hline R-Square & 0.844 & 0.904 & 0.97 \\
\hline Variance & 248.9397 & 198.2908 & 107.1841 \\
\hline Bias & -34.6892 & -26.9142 & -0.02108 \\
\hline M.S.E. & 60871.21 & 38570.78 & 11680.07 \\
\hline R.M.P.S.E & 251.345 & 200.109 & 107.1841 \\
\hline
\end{tabular}

\begin{tabular}{|l|l|l|l|}
\hline DS 4 & Model I & Model II & Model III \\
\hline R-Square & 0.948 & 0.973 & 0.978 \\
\hline Variance & 256.5966 & 188.1626 & 165.7776 \\
\hline Bias & -23.2893 & -13.7181 & -0.01339 \\
\hline M.S.E. & 65009.04 & 34950.9 & 26896.72 \\
\hline R.M.P.S.E & 257.6513 & 188.662 & 165.7776 \\
\hline \multicolumn{5}{|l|}{} \\
\hline DS 5 & Model I & Model II & Model III \\
\hline R-Square & 0.915 & 0.955 & 0.985 \\
\hline Variance & 1063.315 & 794.52 & 444.7486 \\
\hline Bias & -120.802 & -84.8996 & -0.04048 \\
\hline M.S.E. & 1106967 & 615980.3 & 197108.6 \\
\hline R.M.P.S.E & 1070.155 & 799.0431 & 444.7486 \\
\hline \multicolumn{5}{|l|}{} & \\
\hline DS 6 & Model I & Model II & Model III \\
\hline R-Square & 0.899 & 0.932 & 0.941 \\
\hline Variance & 25.59795 & 1.420512 & 30.60204 \\
\hline Bias & -1.45071 & -0.1144 & 3.074886 \\
\hline M.S.E. & 645.8945 & 1.989053 & 923.1052 \\
\hline R.M.P.S.E & 25.63903 & 1.425111 & 30.75614 \\
\hline
\end{tabular}

\subsection{Graphs}

Graphically data are analysed by plotting the actual and predicted values of all three models and the results are very promising. Graphs of all datasets for different models have been shown below from Figure 1-6. 
International Journal of Mathematical, Engineering and Management Sciences

Vol. 4, No. 6, 1508-1521, 2019

https://dx.doi.org/10.33889/IJMEMS.2019.4.6-119

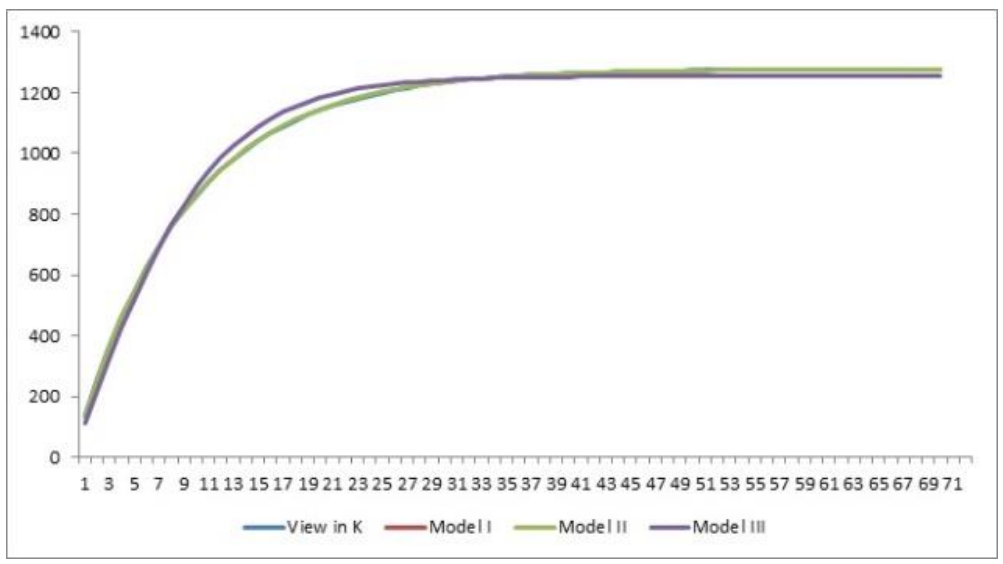

Figure 1. Goodness of fit curve of Dataset 1

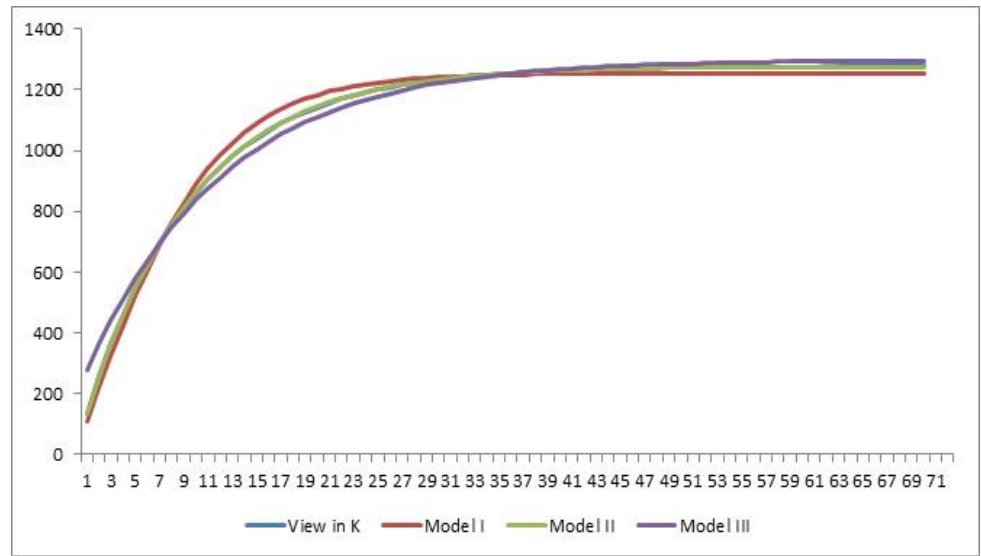

Figure 2. Goodness of fit curve of Dataset 2

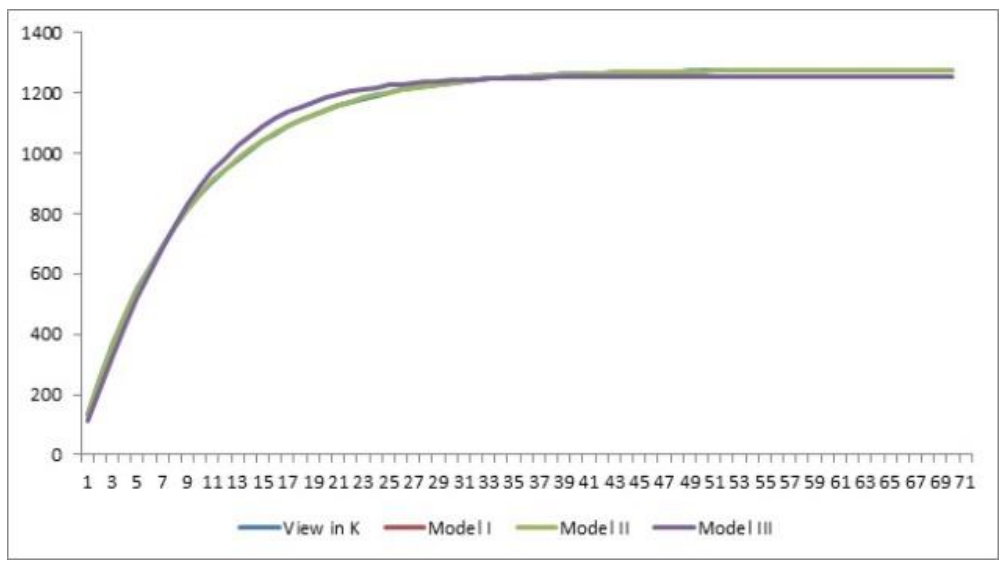

Figure 3. Goodness of fit curve of Dataset 3 
International Journal of Mathematical, Engineering and Management Sciences

Vol. 4, No. 6, 1508-1521, 2019

https://dx.doi.org/10.33889/IJMEMS.2019.4.6-119

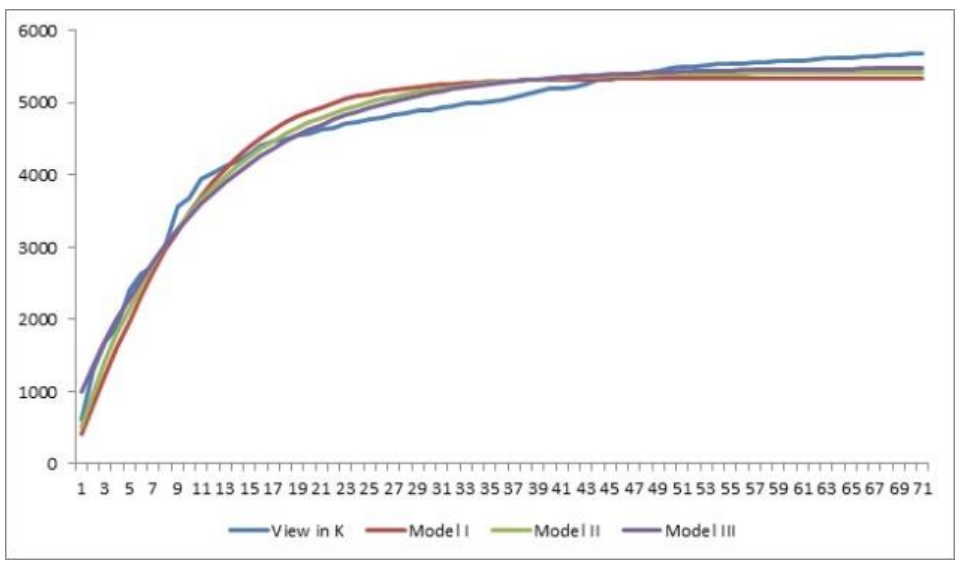

Figure 4. Goodness of fit curve of Dataset 4

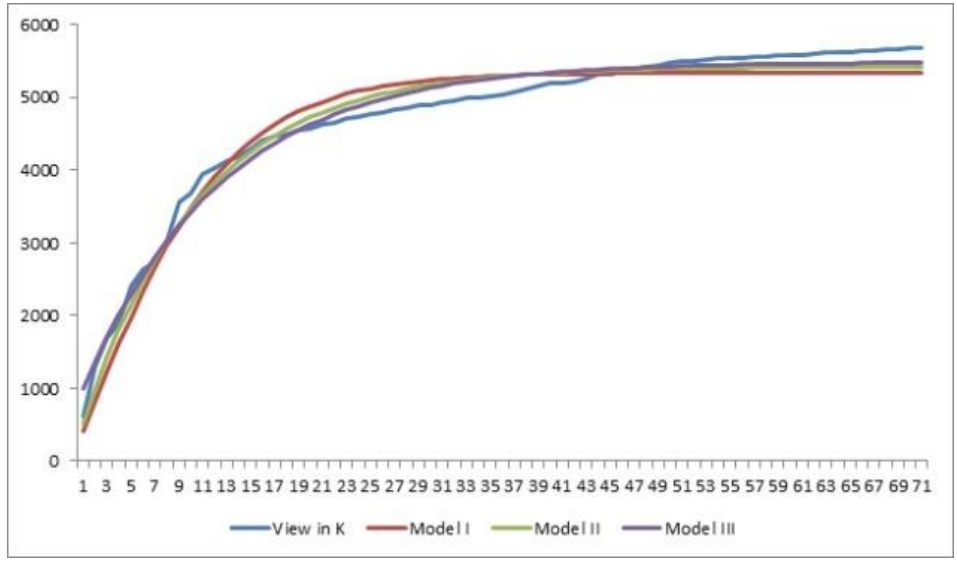

Figure 5. Goodness of fit curve of Dataset 5

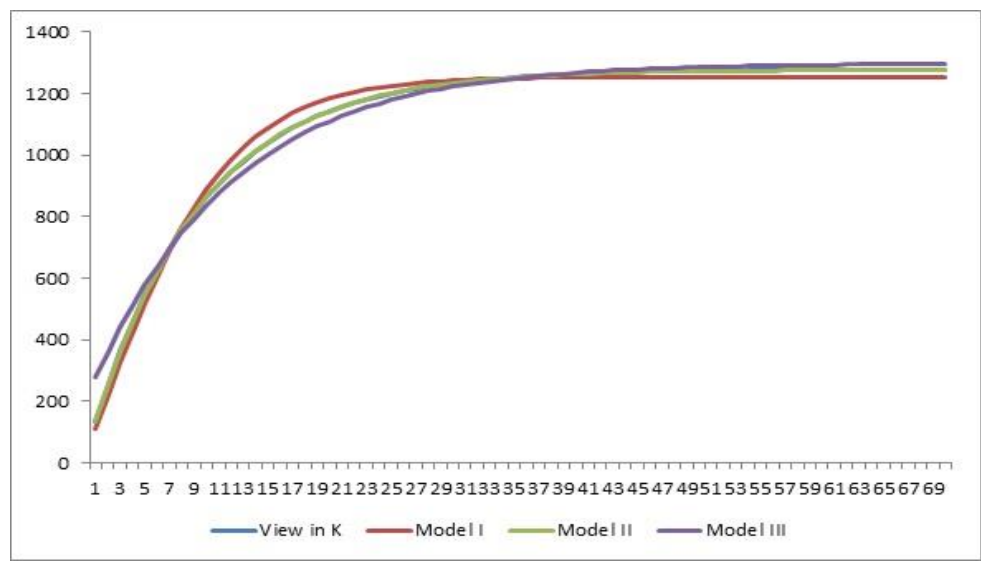

Figure 6. Goodness of fit curve of Dataset 6 
International Journal of Mathematical, Engineering and Management Sciences

Vol. 4, No. 6, 1508-1521, 2019

https://dx.doi.org/10.33889/IJMEMS.2019.4.6-119

\section{Conclusions}

Predicting the view-count is an essential part for knowing the average revenue, internet traffic, what to cache on proxy server for YouTube. Whereas it provides a glimpse of the time window when the return on investment is maximum for the advertiser. In this paper we propose three models for estimating the popularity dynamics of the video and predict the view-count. All three models are derived using different ideologies. Each model caters the impact of different factors on view-count of a video. Model III caters the scenario when the viewership rate of video changes at any time point because of various factors like word of mouth, social impact etc. Model II show subscriptions help in increasing the view-count of the video. Potential market increases with increase of the subscribers of a channel. Number of subscribers may not help in already uploaded videos but provide a good platform for new videos. From Table 5 we can see that on all the performance criteria Model III is describing the data best for all six data sets. From which we can easily state that the initial view-count growth of a video is caused by the subscribers of the channel and after initial hike it view-count rate changes which depends on various external factors. Model II underlies the importance of subscribers in the view-count as it is depicting better results from Model I where we consider diffusion through external factors as the cause of viewcount.

\section{Conflict of Interest}

Authors have no conflict of interest.

\section{Acknowledgements}

The authors would like to thank the reviewers and Editor-in-Chief for carrying out the whole process.

\section{References}

Aggrawal, N., Arora, A., \& Anand, A. (2018a). Modeling and characterizing viewers of You Tube 380 videos. International Journal of System Assurance Engineering and Management, 9(2), 539-546

Aggrawal, N., Arora, A., Anand, A., \& Irshad, M.S. (2018b). View-count based modeling for YouTube videos and weighted criteria-based ranking. In Ram, M., Davim, J.P. (eds) Advanced Mathematical Techniques in Engineering Sciences (pp. 149-160). CRC Press- Taylor and Francis Group, Boca Raton, USA.

Anand, A., \& Bansal, G. (2016). Predicting customer's satisfaction (dissatisfaction) using logistic regression. International Journal of Mathematical, Engineering and Management Sciences, 1(2), 7788 .

Bauckhage, C., Hadiji, F., \& Kersting, K. (2015, April). How viral are viral videos? In Ninth International AAAI Conference on Web and Social Media.

Cheng, X., Dale, C., \& Liu, J. (2008, June). Statistics and social network of youtube videos. In 2008 16th International Workshop on Quality of Service (pp. 229-238). IEEE. Enschede, Netherlands.

Khan, G.F., \& Vong, S. (2014). Virality over YouTube: an empirical analysis. Internet Research, 24(5), 629-647. 
International Journal of Mathematical, Engineering and Management Sciences

Vol. 4, No. 6, 1508-1521, 2019

https://dx.doi.org/10.33889/IJMEMS.2019.4.6-119

Portilla, Y., Reiffers, A., Altman, E., \& El-Azouzi, R. (2015, December). A Study of YouTube recommendation graph based on measurements and stochastic tools. In 2015 IEEE/ACM 8th International Conference on Utility and Cloud Computing (UCC) (pp. 430-435). IEEE. Limassol, Cyprus.

Richier, C., Altman, E., Elazouzi, R., Altman, T., Linares, G., \& Portilla, Y. (2014). Modelling view-count dynamics in youtube. arXiv preprint arXiv:1404.2570.

Singh, V.B., Kapur, P.K., \& Basirzadeh, M. (2010). Instructions executed dependent software reliability growth modeling for open source software by considering change-point. In Proceedings of the 4th National Conference, INDIACom Computing For Nation Development. New Delhi, India.

Yu, H., Xie, L., \& Sanner, S. (2015, April). The lifecyle of a youtube video: phases, content and popularity. In Ninth International AAAI Conference on Web and Social Media. Association for the Advancement of Artificial Intelligence (www.aaai.org).

Zhou, R., Khemmarat, S., \& Gao, L. (2010, November). The impact of YouTube recommendation system on video views. In Proceedings of the 10th ACM SIGCOMM conference on Internet measurement (pp. 404-410). ACM. Melbourne, Australia. 